\title{
Testing for differences in beta diversity from plot-to-plot dissimilarities
}

\begin{abstract}
The central role of beta diversity in describing biodiversity patterns has been investigated in many fields of ecology and biogeography. While a variety of measures of beta diversity have been proposed over the past five decades, the question of how to test for differences in beta diversity among different sets of sampling plots has been addressed only rarely. Here, we describe a simple analytical procedure to test for differences in beta diversity among distinct sets of plots. The advantage of this approach compared to methods that have been previously proposed lies in its randomization procedure. Such a procedure creates a distribution of null values of the test statistic that is compatible with the null hypothesis of no significant difference in multivariate dispersion between the groups. The proposed test was illustrated using a large dataset of plant and water beetle (Coleoptera) assemblages collected from 45 farmland ponds in Ireland.
\end{abstract}

Keywords ANOVA · Farmland ponds · Ireland · Mantel randomization · Multivariate dispersion · Permutation test

G. Bacaro $(\bowtie)$

BIOCONNET, Biodiversity and Conservation Network, Department of Environmental Science "G. Sarfatti",

University of Siena, Via P. A. Mattioli 4, 53100 Siena, Italy

E-mail: bacaro@unisi.it

M. Gioria

School of Agriculture, Food Science and Veterinary Medicine,

University College Dublin, Belfield, Dublin 4, Ireland

E-mail: margheritagioria@yahoo.com

M. Gioria

Ecology, Department of Biology, University of Konstanz,

78457 Konstanz, Germany

\section{Ricotta}

Department of Plant Biology, University of Rome 'La Sapienza', Piazzale Aldo Moro 5, 00185 Rome, Italy

E-mail: carlo.ricotta@uniroma1.it

\section{Introduction}

Beta diversity, defined as the variability in community structure and composition among sites or sampling plots (Whittaker 1960, 1972; Legendre et al. 2005), represents a fundamental concept in community ecology since it provides some insight into the potential factors that make species assemblages more or less similar to one another at different places and times (Anderson et al. 2011; Vellend 2010). The concept of beta-diversity plays a central role in biodiversity assessments and conservation, since it complements information on alpha-diversity, i.e., the number of species at a site, by providing information on the spatial and/or temporal variation in species composition (Margules and Pressey 2000).

The search for the measures of beta diversity that best describe patterns of biodiversity in space and time has long been ongoing. Given the broad generality of this question, since the seminal work of Whittaker (1960, 1972) many different measures of beta diversity have been proposed based on different ecological perspectives and viewpoints (e.g., Wilson and Shmida 1984; Koleff et al. 2003; Legendre et al. 2005; Jost et al. 2010; Anderson et al. 2011; Tuomisto 2010a, b). A number of approaches have been proposed to measure beta diversity, including (1) methods that compute diversity indices for each site and test hypotheses about the factors that may explain the variation among sites (Lande 1996; Veech et al. 2002), or (2) methods that calculate beta diversity by using variation partitioning (Legendre 2008). Methods that measure beta-diversity can also be distinguished in univariate and multivariate measures, depending on whether they refer to patterns in species richness or whether they account for information on species identity and abundance (Anderson 2006; Anderson et al. 2011). In a recent review paper on measures of beta diversity, Anderson et al. (2011) describes two types of measures of $\beta$ diversity: turnover and variation. While turnover aims at measuring changes in community structure between sampling units 
along spatial, temporal, and/or environmental gradients, thus requiring defining specific gradients of interest with directionality, variation in community structure between sample units within a given spatial or temporal context, within a certain group (such as a habitat type or experimental treatment) is measured without having to define any particular gradient or direction. In a multivariate context, this has a direct correspondence with the multivariate dispersion or variance in community structure defined by Anderson (2006) and Anderson et al. (2006). From this latter point of view, a fundamental concept in the analysis of multivariate data is the extent to which groups of observations may differ in their relative dispersions. For example, increases or decreases in the multivariate dispersion of species data can be an important symptom of environmental stress (see Warwick and Clarke 1993; Clarke 1993).

Despite the existing arsenal of metrics that have been proposed over the past five decades, the question of how to test for differences in beta diversity among different sets of sampling plots has been addressed only rarely. Moreover, the majority of the available measures summarize beta diversity by means of a single scalar value, thus they are not appropriate to test for differences in multivariate dispersions among groups of plots.

Anderson (2006) first proposed a test for differences in beta diversity among different groups of plots (PERMDISP), which is essentially a multivariate extension of Levene's test (Levene 1960). Based on Whittaker's (1972) idea that a pairwise plot-to-plot dissimilarity matrix is per se an expression of beta diversity, Anderson (2006) proposed to test for differences in beta diversity among distinct groups of plots calculating an $F$-statistic to compare the average distance of individual plots to their group centroid; a $P$ value is then obtained by permuting such distances among the groups of plots. This method is flexible, since it allows the similarity between pairs of samples to be calculated using any distance/dissimilarity measure of choice. However, while for Euclidean distances, the group centroid can be easily calculated as the arithmetic mean for each variable in the multi-species space, for many ecologically relevant dissimilarity measures like the Jaccard or Bray-Curtis dissimilarities that are not Euclidean embeddable, the calculation of the centroid is not as simple. More specifically, a matrix of $\mathrm{S} \times \mathrm{S}$ plot-to-plot dissimilarities with elements $\mathrm{d}_{i j}$ is said to be Euclidean if the $\mathrm{S}$ plots can be embedded in an Euclidean space such that the Euclidean distance between plot $i$ and plot $j$ is $\mathrm{d}_{i j}$ (Gower and Legendre 1986).

When non-Euclidean dissimilarities are preferred over Euclidean distance measures, principal coordinate analysis (PCoA) can be used to place the plots into a Euclidean space that preserves the original dissimilarities calculated among them. PCoA is an ordination method that takes a symmetric matrix of plot-to-plot dissimilarities and outputs a set of Euclidean coordinates for each plot such that the Euclidean distance between two plots in the full-dimensional principal coordinate space is equal to the original dissimilarity value between both plots (Gower 1966; Legendre and Anderson 1999). If the original dissimilarity measure is Euclidean embeddable, the principal coordinate space contains real axes only. However, if the original dissimilarity is not Euclidean, then the ordination space is composed of both real and imaginary axes, making the underlying calculations more complex (see Anderson 2006).

To overcome these mathematical difficulties, Ricotta and Burrascano (2009) proposed the calculation of an Fstatistic to compare the average distance between individual plots to the pooled set of plots within each group. As in PERMDISP, the $P$ values are then obtained by permuting such distances among groups. Unfortunately, both tests suffer from using an overly simplistic randomization procedure. For instance, given $k$ distinct groups of plots, the null hypothesis to be tested is that there is no significant difference in multivariate dispersion (or beta diversity) between the $k$ groups. Since the value of the multivariate $F$-statistic used to test the null hypothesis is unknown, a distribution of $F$ under the null hypothesis is generated by randomly shuffling the plots into different groups a large number of times. The problem here derives from the fact that, by randomly reshuffling plots among groups, the distances of each plot from the pooled set of plots within each group (in the Ricotta and Burrascano test) or from the group centroid (in PERMDISP) actually change at each randomization step, while in both tests these distances are kept constant during the randomization process. Therefore, this randomization process, for both tests, is not fully compatible with the null hypothesis to be tested.

Here we propose a method to test for differences in beta diversity among distinct groups of plots based on a randomization procedure compatible with the null hypothesis of no significant difference in multivariate dispersion between the groups. To show how the proposed test works in practice, we used data from water beetle and plant communities (species richness and composition) in 45 farmland ponds in Ireland. Computations were performed using the $\mathrm{R}$ software and the codes specifically developed to implement this procedure are presented in "Appendix I".

\section{Description of the test}

To make the test as general and flexible as possible, it is based on any pairwise plot-to-plot dissimilarity matrix of choice that is reasonable for analyzing specific aspects of ecological data. These dissimilarities (not necessarily fulfilling the distance axioms) can be calculated using species presence and absence data, species abundances, or any type of ecologically meaningful functional, morphological or phylogenetic difference between species in different plots (Ives and Helmus 2010; Izsák and Papp 1995; Izsák and Price 2001; Nipperess et al. 2010; Ricotta and Bacaro 2010). 


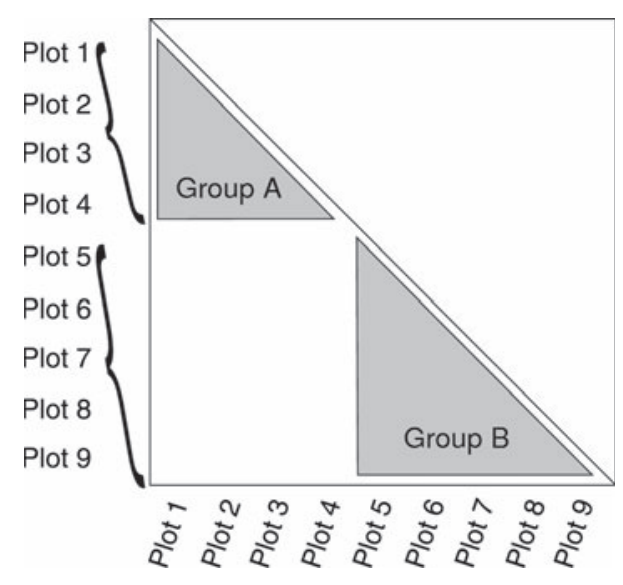

Fig. 1 Schematic example of the proposed test for differences in beta diversity between two groups of plots, $\mathrm{A}$ and $\mathrm{B}$, containing 4 and 5 plots, respectively. First, a dissimilarity matrix is computed among all pairs of plots in both groups. The mean values of the plot-to-plot dissimilarities within each group in the lower halfmatrix are then compared with ANOVA and a $P$ value is calculated by a Mantel randomization of the dissimilarity matrix

Given such a pairwise dissimilarity matrix, the beta diversity of a certain group of plots can be defined as the mean of the plot-to-plot dissimilarities within the group (Legendre et al. 2005; Whittaker 1972). According to this definition, a test for differences in beta diversity among different groups of plots can be performed simply by comparing with ANOVA the mean of these plot-to-plot dissimilarities among groups. A distribution of values of the test-statistic under the null hypothesis is then obtained by Mantel randomization of the dissimilarity matrix (see Fig. 1 for a schematic example).

\section{A case study}

To illustrate the proposed test with a real dataset, we used data from farmland ponds in Ireland sampled specifically to investigate the strength of vascular plant species composition in predicting the composition of water beetle (Coleoptera) assemblages (Gioria et al. 2010a, 2011). Farmland ponds play a major role in maintaining biodiversity at the regional/landscape level, and may support more species, as well as more uncommon, rare, and threatened species, compared to other freshwater ecosystem types (see Gioria et al. $2010 \mathrm{a}, \mathrm{b}$ and references therein). In Ireland, farmland ponds support a large proportion of the overall water beetle fauna as well as species listed in the IUCN Red List of Irish Water Beetles (Foster et al. 2009). Here, we analyze a subset of this entire dataset (Gioria et al. 2010a) comprising 45 permanent ponds from which 67 plant and 77 water beetle species were recorded. The ponds used for this study were sampled in two regions (Region 1, Wexford, 19 ponds; $52^{\circ} 23^{\prime} \mathrm{N}, 6^{\circ} 23^{\prime} \mathrm{W}$; Region 2, Mullingar, 26 ponds; $53^{\circ} 33^{\prime} \mathrm{N}, 7^{\circ} 25^{\prime} \mathrm{W}$ ). Plant data are expressed as percentage cover over a sampling area comprised within $2 \mathrm{~m}$ from the pond margin within each microhabitat type. Beetle abundance data are expressed as beetle counts collected over a standardized time (3-min 'net-in-the-water', with sampling time divided equally across microhabitat type) within the sampling area where plant data were recorded. This protocol allowed a direct comparison of plant and beetle data since they were collected over the same surface area (Gioria et al. 2011). Samples were collected in June/July 2008 for ponds located in Region 1, and in June/July 2009 for ponds located in Region 2.

A relationship between plants and water beetles was expected. Gioria et al. (2010a, b, 2011) showed that there is a moderate, consistent correlation in species composition and diversity between plants and water beetles. The nature of the relationship between these taxonomic groups has long been described. Patterns in water beetle composition are known to be correlated to the structure and composition of the vegetation (e.g., Ranta 1985; Friday 1987; Gee et al. 1997; Painter 1999; Declerck et al. 2006). Foster et al. (1992) showed that the abundance of emergent vegetation was among the most important factors in classifying Irish water beetles. Foster and Eyre (1992) also suggested that the density of the vegetation in standing water was important in determining patterns in water beetle assemblages in Britain. Gioria et al. (2010a, b) suggested that the significant contribution of plant community composition in explaining patterns in water beetle assemblages could depend upon a combination of factors. First, the composition of wetland plants directly affects the characteristics of the micro-habitats available to water beetles by determining the physical structure of the vegetation at a locality as well as the availability of oviposition sites, food sources, and shelter from predators (see also Painter 1999). Morover, the composition of the vegetation has a direct effect on the stability of the substrate and the cycling of nutrients (e.g., Keddy 2000). Second, the composition of the vegetation incorporates information on the biotic and abiotic conditions at a locality (Keddy 2000; Declerck et al. 2006; Schaffers et al. 2008). For instance, the presence of submerged plant species incorporates information on pond permanency and minimum depth, whilst, for instance, the dominance of grasses and rushes encompasses information on pond permanency and/or grazing intensity.

Plot-to-plot dissimilarity matrices were calculated using (1) the Bray and Curtis (1957) dissimilarity measure, based on plants and beetle abundance data, and (2) the Jaccard (1900) distance based on presence/absence only. Differences in beta diversity between plant and beetle assemblages were tested for the whole set of ponds and for each region separately by comparing the average of the calculated dissimilarities between the two groups (plants and beetles) using an $F$ test. $P$ values were computed from 999 permutations of the plot-to-plot dissimilarities between the two groups. All calculations were performed running a new function ('BetaDispersion' 
freely available in "Appendix I") within the R statistical environment (R Development Core Team 2010).

\section{Results}

The mean beta diversity values for each taxonomic group are presented in Table 1. For the whole set of ponds as well as for each region, the average pairwise dissimilarities were higher for plant assemblages than for beetle assemblages. The differences in the beta diversity between plants and beetles, tested using the permutational procedure here described, were significantly different, for the whole dataset and within each region ( $P$ values $=0.001$ for all the performed comparisons, Table 1).

\section{Discussion}

The dissimilarity-based test here described represents a straightforward way of exploring differences in multivariate dispersions or beta diversity among groups of plots. While a number of different approaches have been proposed to test for differences in beta diversity among groups of plots (Anderson 2006; Ricotta and Burrascano 2009), all testing procedures developed to date are based on (1) a metric measuring the actual differences in beta diversity among distinct sets of plots, and (2) a randomization algorithm to assess the distribution of the metric under a given null model. However, defining an adequate null model for the specific hypothesis to be tested is not a simple operation as slightly different randomization procedures may imply considerably diverse null hypotheses that can ultimately have subtle and often unintended effects on the actual ecological hypotheses to be tested (Fortin and Jacquez 2000). Being based on Mantel randomization of the original plot-toplot dissimilarity matrix, the test proposed in this paper seems indeed compatible with the null hypothesis of no significant difference in multivariate dispersion between groups of plots.
The results of this study, based on field data, show that the proposed method is useful to characterize differences in beta diversity between different taxonomic groups. From an ecological point of view, our results showed that, in 45 permanent ponds in Ireland, the average plant beta diversity was consistently higher than that of water beetles. This was evident both when analyzing the full dataset and the regional datasets.

Both theoretical and empirical studies have shown that organisms usually show a spatial distribution pattern if essential components of their environment are patterned (Capers et al. 2010). Exogenous factors, such as climate, soil type or solar activity, may regulate species distribution and diversity patterns at the global scale based on spatial autocorrelation, related to a similar occurrence probability in neighboring sites, simply because the external factors show a specific autocorrelation pattern (Dormann 2006). At a finer spatial scale, biotic and abiotic processes may further cause a structuring within smaller areas of relative environmental homogeneity or heterogeneity, provoking a small-scale niche limitation (Legendre 1993; Wagner 2003). Thus, species may appear and disappear along a compositional gradient (Wagner 2003; Bacaro and Ricotta 2007) at several spatial scales, creating patterns of beta diversity (or beta similarity). Moreover, the concept of beta diversity can be applied along time intervals for the same community. This is relevant when sampling communities that exhibit temporary pulses. In fact comparison among points will yield results that are largely dependent on individual pulse phases of each community.

With respect to the dataset analyzed, highlighted differences in beta diversities likely reflect the contribution of the main drivers of plant and water beetle diversity patterns in Irish farmland ponds: among such factors is pond size and depth.

While small ponds tend to be characterized by a swamp zone dominated by Typha latifolia and/or Phragmites australis, larger ponds tend to support

Table 1 Analyses of variance testing for significant differences in beta diversity between plant and beetle assemblages

\begin{tabular}{|c|c|c|c|c|c|}
\hline Dataset & Dissimilarity measure & $\begin{array}{l}\text { Average beta } \\
\text { diversity } \\
\text { Beetles }\end{array}$ & $\begin{array}{l}\text { Average beta } \\
\text { diversity } \\
\text { Plants }\end{array}$ & $F$ Model & $P$ value \\
\hline \multirow[t]{2}{*}{ Full dataset } & Bray-Curtis & 0.696 & 0.807 & 239.39 & 0.001 \\
\hline & Jaccard & 0.708 & 0.831 & 562.61 & 0.001 \\
\hline \multirow[t]{2}{*}{ Region 1} & Bray-Curtis & 0.529 & 0.670 & 85.11 & 0.001 \\
\hline & Jaccard & 0.623 & 0.741 & 112.43 & 0.001 \\
\hline \multirow[t]{2}{*}{ Region 2} & Bray-Curtis & 0.684 & 0.819 & 96.54 & 0.001 \\
\hline & Jaccard & 0.686 & 0.820 & 165.20 & 0.001 \\
\hline
\end{tabular}

Two measures of dissimilarity were used to calculate beta diversity: the Bray-Curtis dissimilarity measure (for species abundance data) and the Jaccard coefficient (for presence/absence data). Analyses were carried out for the whole set of 45 ponds as well as for each region separately (Region 1, 19 ponds; Region 2, 26 ponds). $P$ values were obtained by Mantel randomization of the original plot-to-plot distance matrices (999 permutations) 
species-rich plant assemblages characterized by a range of emergent, floating-leaved, as well as submerged species (Gioria et al. 2010a, b). On the contrary, the composition of water beetle assemblages is less affected by differences in these variables (Gioria et al. 2010a, b and references therein).

In a similar fashion, grazing also has a major effect on vegetation, leading to communities dominated by Juncus species, grasses and zones of bare substratum (Gioria et al. 2010b). The dominant substratum (clay vs gravel) also substantially affects the diversity and composition of plant assemblages, with gravel ponds typically richer than those dominated by mud/clay. This was evident in the fact that the dissimilarity calculated for plant assemblages was higher in Region 2, where seven ponds were dominated by a gravel substratum compared to only two found in Region 1 (see Gioria et al. 2010a). The range of $\mathrm{pH}$ was also greater in Region 2, where a number of ponds supported charophyte species. On the other hand, a lower beta diversity calculated for water beetles can be due partly to the fact that those assemblages included a 'core' of common species, widespread and generally abundant in Ireland, that was found at each pond, independently of pond size, management regime, or dominant substratum. However, since different environmental factors operate at different spatial scales, patterns of beta diversity should depend on the grain size at which they are analyzed (Steinitz et al. 2006). Similarly, extent is expected to influence the pattern of species complementarity, since increasing extent should result in (1) a higher habitat heterogeneity, and (2) a higher probability to include geographical barriers in the area considered.

From a more general viewpoint, the possibility to use any meaningful dissimilarity coefficient for summarizing beta diversity makes our method flexible, in line with the broad definition of beta diversity used in this paper. As these measures summarize plot-to-plot dissimilarity from different viewpoints and perspectives, the most important methodological decision consists of selecting the coefficient that best captures the specific aspect of plot-to-plot dissimilarity we want to measure. Nonetheless, the same decision has to be made in virtually any field of multivariate analysis. Therefore, we see this generality more as an advantage than as a disadvantage, allowing ecologists to compute relevant facets of beta diversity with the largest possible flexibility.

Acknowledgments Part of this work was done by G.B. during a visiting research period at the Institute of Hazard, Risk and Resilience, Department of Geography, University of Durham (UK), founded by the "Luigi and Francesca Brusarosco" Foundation. Insightful comments and suggestions by the Editor Prof. Brook and two anonymous referees on a previous draft of this paper are also warmly acknowledged.

\section{Appendix I: R function 'BetaDispersion'}

This program is free software: you can redistribute it and/or modify it under the terms of the GNU General Public License http://www.gnu.org/licenses/.

Disclaimer Users of this code are cautioned that, while due care has been taken and it is believed accurate, it has not been rigorously tested and its use and results are solely the responsibilities of the user.

Description The function 'BetaDispersion' performs test for differences in beta diversity among distinct groups of plots. The test is based on any pairwise plotto-plot dissimilarity matrix of choice that is reasonable for analyzing specific aspects of ecological data. Given such a pairwise dissimilarity matrix, BetaDispersion compares with ANOVA the mean of the plot-to-plot dissimilarities among groups. A distribution of values of the test-statistic under the null hypothesis is then obtained by Mantel randomization of the dissimilarity matrix.

\section{Dependencies None} 999)

Usage Betadispersion(data, factor, permutations =

\section{Arguments}

Data An $n \times n$ matrix of distances or an object of class "dist" (as obtained by functions as vegdist or gowdis).

Factor Vector describing the group structure, usually a factor or an object that can be coerced to a factor using 'as.factor'.

Permutations Number of replicate permutations used for the hypothesis tests ( $F$ tests). If not provided, the default value of 999 is assumed.

\section{Outputs}

The function returns a list of two objects:

1. 'Anova table': typical analysis of variance table showing sources of variation, degrees of freedom, sequential sums squares, mean squares, $F$ statistics and $P$ value, based on the selected number of permutations.

2. Mean within group distance.

Details Significance tests are done using $F$-tests based on sequential sums of squares from permutations of the raw data, and not permutations of residuals. Permutations of the raw data may have better small sample characteristics. Further, the precise meaning of hypothesis tests will depend upon precisely what is permuted. 
Function syntax

betadispersion $<$-function(data, factor, permutation)

\{

$\mathrm{x}<$-as.dist(data)

$\mathrm{x}$.estend $<-$ as.vector $(\mathrm{x})$

if (missing(permutation))

permutation $<-999$

$\mathrm{N}<-$ attributes(x)\$Size

grouping $<-$ as.factor(factor)

matched $<$ - function(irow, icol, grouping) \{

grouping[irow $]==$ grouping $[\mathrm{icol}]$

\}

irow $<$ - as.vector(as.dist $(\operatorname{row}(\operatorname{matrix}($ nrow $=\mathrm{N}$, ncol $=\mathrm{N})))$ )

icol $<-$ as.vector $(\operatorname{as} \cdot \operatorname{dist}(\operatorname{col}(\operatorname{matrix}($ nrow $=\mathrm{N}$, ncol $=\mathrm{N})))$ )

within $<-$ matched(irow, icol, grouping)

cl.vec $<-$ rep("Between", length $(\mathrm{x})$ )

take $<-$ as.numeric(irow[within])

cl.vec[within] <- levels(grouping)[grouping[take]]

cl.vec $<-$ factor(cl.vec, levels = c("Between", levels(grouping)))

frame $<$-data.frame(x.estend, cl.vec)

reduced $<$-frame[!cl.vec $==$ "Between", ]

oneway $<-\operatorname{lm}(\mathrm{x}$. estend $\sim$ cl.vec, data $=$ reduced $)$

promo $<$-anova(oneway, test $=$ "F")

F.mod $<$-promo[1,4]

Average_Beta_Whitin $<-$ tapply(reduced\$x.estend, list(cl.vec $=$ reduced\$cl.vec), mean, na.rm $=$ TRUE)

permutedist $<$ - function $(\mathrm{m})\{$

permutevec $<$ - function(v, perm) return(v[perm])

$\mathrm{m}<-$ as.matrix $(\mathrm{m})$

$\mathrm{n}<-\operatorname{ncol}(\mathrm{m})$

w0 $<-$ sample(n)

mperm <- apply $(\mathrm{m}, 1$, permutevec, perm $=$ w0)

mperm $<-\mathrm{t}($ mperm $)$

mperm <-apply(mperm, 2, permutevec, perm = w0)

return(as.dist $(\mathrm{t}($ mperm $)))$

\}

permF $<$-array $(\operatorname{dim}=\mathrm{c}($ permutation, 1$))$

for (i in 1:permutation)

\{

permi $<$-permutedist $(\mathrm{x})$

vect $<-$ as.vector(permi)

asso $<$-data.frame(vect, cl.vec)

reduced_asso $<$-asso[!cl.vec $==$ "Between", ]

model $<-\operatorname{lm}($ vect $\sim$ cl.vec, data $=$ reduced_asso $)$

vin $<-$ anova(model,test $=$ "F")

permF $[i, 1]<-$ vin $[1,4]$

\}

tab $<$ - data.frame(Df $=$ promo[,1], SumsOfSqs $=$ promo[,2],

MeanSqs $=$ promo[,3], F.Model $=$ promo[,4],

$\mathrm{P}=\mathrm{c}((\operatorname{rowSums}(\mathrm{t}($ permF $)>=$ F.mod $)+1) /($ permutation +

1), NA))

rownames(tab) <- c("Factor",,'Residuals")

colnames(tab) [ncol(tab)] <- "Pr( $>$ F)"

class(tab) <- c("anova", class(tab))

Average_Beta_Whitin $<$-data.frame(Average_Beta_Whitin)

coll <-row.names(Average_Beta_Whitin)

col $<<$-Average_Beta_Whitin[,1]

Summary_beta $<$-data.frame(col1,col2)

Summary $<$-data.frame(Summary_beta[2:nrow(Summary_beta),]) 
colnames(Summary) <-c(“Group", “Average Beta”) output $<-$-list(tab,Summary)

output

\}

Example (require the "vegan" library)

data(dune)

data(dune.env)

dune.dist $<$ - vegdist(dune)

$\operatorname{attach}($ dune.env)

betadispersion(dune.dist, Management,999)

\section{References}

Anderson MJ (2006) Distance-based tests for homogeneity of multivariate dispersions. Biometrics 62:245-253

Anderson MJ, Ellingsen KE, McArdle BH (2006) Multivariate dispersion as a measure of beta diversity. Ecol Lett 9:683-693

Anderson MJ, Crist TO, Chase JM, Vellend M, Inouye BD, Freestone AL, Sanders NJ, Cornell HV, Comita LS, Davies KF, Harrison SP, Kraft NJB, Stegen JC, Swenson NG (2011) Navigating the multiple meanings of beta diversity: a road map for the practicing ecologist. Ecol Lett 14:19-28

Bacaro G, Ricotta C (2007) A spatially explicit measure of beta diversity. Community Ecol 8:41-46

Bray JR, Curtis JT (1957) An ordination of the upland forest communities of southern Wisconsin. Ecol Monogr 27:325349

Capers RS, Selsky R, Bugbee GJ (2010) The relative importance of local conditions and regional processes in structuring aquatic plant communities. Freshw Biol 55:952-966

Clarke K (1993) Non-parametric multivariate analyses of changes in community structure. Aust J Ecol 18:117-143

Declerck S, De Bie T, Ercken D, Hampel H, Schrijvers S, Van Wichelend J, Gillard V, Mandiki R, Losson R, Bauwens D, Keijers S, Vyverman W, Goddeeris B, De meester L, Brendonck L, Martens K (2006) Ecological characteristics of small farmland ponds: associations with land use practices at multiple spatial scales. Biol Conserv 131:523-532

Dormann CF (2006) Effects of incorporating spatial autocorrelation into the analysis of species distribution data. Glob Ecol Biogeogr 16:129-138

Fortin MJ, Jacquez GM (2000) Randomization tests and spatially autocorrelated data. ESA Bull 81:201-205

Foster GN, Eyre MD (1992) Classification ranking of water beetle communities. UK nature conservation, vol 1. Joint Nature Conservation Committee, Peterborough

Foster GN, Nelson BH, Bilton DT, Lott DA, Merritt R, Weyl RS, Eyre MD (1992) A classification and evaluation of Irish water beetle assemblages. Aquat Conserv Mar Freshw Ecosyst 2:185-208

Foster GN, Nelson BH, O'Connor O (2009) Ireland Red List no. 1 -water beetles. National Parks and Wildlife Service, Department of Environment, Heritage and Local Government, Dublin

Friday LE (1987) The diversity of macroinvertebrate and macrophyte communities in ponds. Freshw Biol 18:87-104

Gee JHR, Smith BD, Lee KM, Griffiths SW (1997) The ecological basis of freshwater pond management for biodiversity. Aquat Conserv Mar Freshw Ecosyst 7:91-104
Gioria M, Schaffers A, Bacaro G, Feehan J (2010a) The conservation value of farmland ponds: predicting water beetle assemblages using vascular plants as a surrogate group. Biol Conserv 143:1125-1133

Gioria M, Bacaro G, Feehan J (2010b) Identifying the drivers of pond biodiversity: the agony of model selection. Community Ecol 11:179-186

Gioria M, Bacaro G, Feehan J (2011) Evaluating and interpreting cross-taxon congruence: potential pitfalls and solutions. Acta Oecol 37:187-194

Gower JC (1966) Some distance properties of latent root and vector methods used in multivariate analysis. Biometrika 53:325-338

Gower JC, Legendre P (1986) Metric and Euclidean properties of dissimilarity coefficients. J Classif 3:5-48

Izsák J, Papp L (1995) Application of the quadratic entropy indices for diversity studies of drosophilid assemblages. Environ Ecol Stat 2:213-224

Izsák C, Price RG (2001) Measuring $\beta$-diversity using a taxonomic similarity index, and its relation to spatial scale. Mar Ecol Prog Ser 215:69-77

Ives AR, Helmus MR (2010) Phylogenetic metrics of community similarity. Am Nat 176:E128-E142

Jost L, DeVries P, Walla T, Greeney H, Chao A, Ricotta C (2010) Partitioning diversity for conservation analyses. Divers Distrib 16:65-76

Keddy PA (2000) Wetland ecology: principles and conservation. Cambridge University Press, Cambridge

Koleff P, Gaston KJ, Lennon JJ (2003) Measuring beta diversity for presence-absence data. J Anim Ecol 72:367-382

Lande R (1996) Statistics and partitioning of species diversity, and similarity among multiple communities. Oikos 76:5-13

Legendre P (1993) Spatial autocorrelation: trouble or new paradigm? Ecology 74:1659-1673

Legendre P (2008) Studying beta diversity: ecological variation partitioning by multiple regression and canonical analysis. J Plant Ecol 1:3-8

Legendre P, Legendre L (1998) Numerical ecology, 2nd edn. Elsevier, Amsterdam

Legendre P, Anderson MJ (1999) Distance-based redundancy analysis: testing multispecies responses in multifactorial ecological experiments. Ecol Monogr 69:1-24

Legendre P, Borcard D, Peres-Neto PR (2005) Analyzing beta diversity: partitioning the spatial variation of community composition data. Ecol Monogr 75:435-450

Levene H (1960) Robust tests for equality of variances. In: Olkin I, Ghurye SG, Hoeffding W, Madow WG, Mann HB (eds) Contributions to probability and statistics. Stanford University Press, Stanford, pp 278-292 
Jaccard P (1900) Contribution au problème de l'immigration postglaciare de la flore alpine. Bull Soc Vaudoise Sci Nat 36:87-130

Margules CR, Pressey RL (2000) Systematic conservation planning. Nature 405:243-253

Nipperess DA, Faith DP, Barton K (2010) Resemblance in phylogenetic diversity among ecological assemblages. J Veg Sci 21:809-820

Painter D (1999) Macroinvertebrate distributions and the conservation value of aquatic Coleoptera, Mollusca, and Odonata in the ditches of traditionally managed and grazing fen at Wicken Fen, UK. J Appl Ecol 36:3-48

R Development Core Team (2010) R: a language and environment for statistical computing. R Foundation for Statistical Computing, Vienna. ISBN: 3-900051-07-0. http://www.R-project. org/

Ranta E (1985) Communities of water beetles in different kinds of water in Finland. Proc Acad Nat Sci Phila 137:33-45

Ricotta C, Bacaro G (2010) On plot-to-plot dissimilarity measures based on species functional traits. Community Ecol 11:113-119

Ricotta C, Burrascano S (2009) Testing for differences in beta diversity with asymmetric dissimilarities. Ecol Indic 9:719-724

Schaffers AP, Raemakers IP, Sýkora KV, ter Braak CJF (2008) Arthropod assemblages are best predicted by plant species composition. Ecology 89:782-794

Steinitz O, Heller J, Tsoar A, Rotem D, Kadmon R (2006) Environment, dispersal and patterns of species similarity. J Biogeogr 33:1044-1054
Tuomisto H (2010a) A diversity of beta diversities: straightening up a concept gone awry. Part 1. Defining beta diversity as a function of alpha and gamma diversity. Ecography 33:2-22

Tuomisto H (2010b) A diversity of beta diversities: straightening up a concept gone awry. Part 2. Quantifying beta diversity and related phenomena. Ecography 33:23-45

Veech J, Summerville K, Crist T, Gering J (2002) The additive partitioning ofspecies diversity: recent revival of an old idea. Oikos 99:3-9

Vellend M (2010) Conceptual synthesis in community ecology. Q Rev Biol 85:183-206

Wagner HH (2003) Spatial covariance in plant communities: integrating ordination, geostatistics, and variance testing. Ecology 84:1045-1057

Warwick RM, Clarke KR (1993) Comparing the severity of disturbance: a meta-analysis of marine macrobenthic community data. Mar Ecol Prog Ser 92:221-231

Whittaker RH (1960) Vegetation of the Siskiyou Mountains, Oregon and California. Ecol Monogr 30:279-338

Whittaker RH (1972) Evolution and measurement of species diversity. Taxon 21:213-251

Wilson MW, Shmida A (1984) Measuring beta diversity with presence-absence data. J Ecol 72:1055-1064 